\title{
K-derivation and symmetric bi-k-derivation on Gamma Banach Algebras
}

\author{
${ }^{1} \mathrm{P}$. Rajkhowa, ${ }^{2} \mathrm{Md}$. Shahidul Islam Khan \\ Department of Mathematics, Gauhati University, Guwahati-781014
}

\begin{abstract}
In this paper, we define and study k-derivations and symmetric bi-k-derivations on a $\Gamma$-Banach algebra. We also define and study $h \otimes k$-derivation $d$ on the projective tensor product $V \otimes_{p} V^{\prime}$ for the $h$-and $k$-derivations $d_{1}$ and $d_{2}$ on $\Gamma$-Banach Algebras $V(F)$ and $V^{\prime}\left(F^{\prime}\right)$ respectively.
\end{abstract}

AMS subject classification Code: $17 D 20(\gamma, \delta)$

Key words: $k$-derivation, symmetric bi-k-derivation, centroid.

\section{Introduction:}

N. Nobusawa [7] introduced the notion of a $\Gamma$-ring, more general than a ring. W. E. Barnes [11] weakened slightly the condition in definition of $\Gamma$-ring in the sense of Nobusawa. W. E. Barnes [11], J. Luh [4] and S. Kyuno [10] studied the structure of $\Gamma$-rings and obtained various generalizations analogous to corresponding parts in ring theory. Bhattacharya and Maity [2] introduced the notion of a $\Gamma$-Banach algebra. In recent times, many far reaching results of general algebras have been extended to $\Gamma$-algebras by many outstanding research workers. In this paper, we study $\mathrm{k}$-derivation on $\Gamma$-Banach algebras $\mathrm{V}$ and $\mathrm{k} \otimes \mathrm{h}$ - deviation on $\Gamma \otimes \Gamma$-Banach algebra $V \otimes_{p} V^{\prime}$ We define symmetric bi-k-derivation on $\Gamma$-Banach algebras in which $\mathrm{k}$ :

$\Gamma \rightarrow \Gamma$ is an additive map such that $k^{n}=k$, where $\mathrm{n}$ is a positive integers. Some important results relating to this concepts are proved. For example we show that (a) Let $V(F)$ and $V^{\prime}\left(F^{\prime}\right)$ be two $\Gamma$-Banach algebra and $\Gamma^{\prime}$-Banach algebra respectively with $e \delta x=x \delta e=x(\forall x \in V), \quad e \in V, \delta \in \Gamma \quad$ and $e^{\prime} \delta^{\prime} y=y \delta^{\prime} e^{\prime}=y(\forall \mathrm{y} \in \mathrm{V}), e^{\prime} \in V^{\prime}, \delta^{\prime} \in \Gamma^{\prime}$. If $\mathrm{d}_{1}$ and $\mathrm{d}_{2}$ are k- and h-inner derivation on $V(F)$ and $V^{\prime}\left(F^{\prime}\right)$ respectively implemented by $(a, \delta)$ and $\left(\mathrm{b}, \delta^{\prime}\right)$ respectively then $\mathrm{d}$ is a $\mathrm{k} \otimes \mathrm{h}$ - inner deviation on $V \otimes_{p} V^{\prime}$ implemented by $\left(\mathrm{a} \otimes \mathrm{e}^{\prime}+\mathrm{e} \otimes \mathrm{b}, \delta \otimes \delta^{\prime}\right)$, (b) Let $\mathrm{V}$ be a 2-torsion free prime $\Gamma$-Banach algebra, $\mathrm{D}_{1}(. .),. \mathrm{D}_{2}(.,$.$) and \mathrm{D}_{3}(. .$.$) and \mathrm{D}_{4}(. .$.$) the symmetric bi-k-derivations on \mathrm{V}$ and $\mathrm{d}_{1}, \mathrm{~d}_{2}, \mathrm{~d}_{3}$ and $\mathrm{d}_{4}$ traces of $\mathrm{D}_{1}(.,),. \mathrm{D}_{2}(.,),. \mathrm{D}_{3}(.,$.$) and \mathrm{D}_{4}(.,$.$) respectively. If \mathrm{d}_{1}(\mathrm{x}) \gamma \mathrm{d}_{2}(\mathrm{y})=\mathrm{d}_{3}(\mathrm{x}) \gamma \mathrm{d}_{4}(\mathrm{y})$, for all $\mathrm{x}, \mathrm{y} \in \mathrm{V}$ and $\gamma \in \Gamma$ and $d_{1} \neq 0 \neq d_{4}$, then there exists $\lambda \in C_{\Gamma}$ such that $d_{2}(x)=\lambda \alpha d_{1}(x)$ for all $\alpha \in \Gamma$, where $C_{\Gamma}$ is the extended centroid of $\mathrm{V}$, (c) Let $\mathrm{V}$ be a 2-torsion free prime Gamma Banach algebra and $\mathrm{U}$ be a non zero ideal of V. Suppose there exist symmetric bi-k-derivations $D_{1}: V \times V \rightarrow V$ and $D_{2}: V \times V \rightarrow V$ such that $\mathrm{D}_{1}\left(\mathrm{~d}_{2}(\mathrm{x}), \mathrm{x}\right)=0$ holds for all $\mathrm{x} \in \mathrm{U}$ where $\mathrm{d}_{2}$ denotes the trace of $\mathrm{D}_{2}$. In this case $\mathrm{D}_{1}=0$ or $\mathrm{D}_{2}=0$, (d) Let $\mathrm{V}$ be a 2- and 3-torsion free prime $\Gamma$-Banach algebra. Let $\mathrm{U}$ be a non zero ideal of $\mathrm{V}$ and $\mathrm{D}_{1}: \mathrm{V} \times \mathrm{V} \rightarrow \mathrm{V}$ and $\mathrm{D}_{2}: \mathrm{V} \times \mathrm{V} \rightarrow \mathrm{V}$ be symmetric bi-k- derivations. Suppose further that there exists a symmetric bi-additive mapping B: $V \times V \rightarrow V$ such that $d_{1}\left(d_{2}(x)\right)=f(x)$ holds, for all $x \in U$, where $D_{1}$ and $D_{2}$ are the traces of $D_{1}$ and $D_{2}$ respectively and $f$ is the trace of $B$. Then either $D_{1}=0$ and $D_{2}=0$.

\section{Preliminaries}

Let $\mathrm{V}$ and $\Gamma$ be two additive abelian groups. If for all $x, y, z \in V ; \gamma, \mu \in \Gamma$, the following conditions are satisfied, (a) $x \gamma y \in V$, (b) $(x+\gamma) \gamma z=x \gamma z+y \gamma z, x(\gamma+\mu) y=x \gamma y+x \mu y$, 
$\mathrm{X} \gamma(\mathrm{y}+\mathrm{z})=\mathrm{X} \gamma \mathrm{y}+\mathrm{X} \gamma \mathrm{z} \quad$ (c) $x \gamma(y \mu z)=(x \gamma y) \mu z$ then $\mathrm{V}$ is called a $\Gamma$ - ring in the sense of

Barnes[11]. If these conditions are strengthened to, $\left(a^{\prime}\right) \quad x \gamma y \in V,\left\{x \mu \in \Gamma,\left(b^{\prime}\right)\right.$ is same as(b),

(c) $(x+\gamma) \gamma z=x \gamma z+y \gamma z, \quad x(\gamma+\mu) y=x \gamma y+x \mu y, \quad x \gamma(y+z)=x \gamma y+x \gamma z$

$x \gamma(y \mu z)=x(\gamma y \mu) z=(x \gamma y) \mu z \quad$ (d) $x \gamma y=0, \forall x, y \in V$ implies $\quad \gamma=0$, Then $\mathrm{V}$ is called a $\Gamma$ ring in the sense of Nobusawa.

A $\Gamma$-ring in the sense of Nobusawa $\mathrm{V}$ is called a $\Gamma$-Banach algebra over a field $\mathrm{F}$ if it satisfies the following postulates:

(a) $\quad \mathrm{a}(\mathrm{x} \gamma \mathrm{y})=(\mathrm{ax}) \gamma \mathrm{y}=\mathrm{x} \gamma(\mathrm{ay}), \mathrm{a} \in \Gamma ; \mathrm{x}, \mathrm{y} \in \mathrm{M} ; \gamma \in \Gamma$.

(b) $\quad \mathrm{M}$ is a Banach space over $\mathrm{F}$ with respect to a norm which satisfies

$\|x \gamma y\| \leq\|x\|\|\gamma\|\|y\|, x, y \in V ; \gamma \in \Gamma$

A subset $\mathrm{I}$ of a $\Gamma$ - Banach algebra $\mathrm{V}$ is said to be a right (left) ideal of $\mathrm{V}$ if

(a) $\quad \mathrm{I}$ is a subspace of $\mathrm{V}$ (in the vector space sense).

(b) $x y y \in I(y \not x \in I)$ for all $x \in I, \gamma \in \Gamma, y \in V$

i.e. $I \Gamma V \subseteq I(V \Gamma I \subseteq I)$

A right $\Gamma$-ideal which is a left $\Gamma$-ideal as well as is called a two sided $\Gamma$-ideal or simply a $\Gamma$ ideal.

The notation $I \triangleleft V$ will mean $\mathrm{I}$ is an ideal of $\mathrm{V}$.

A $\Gamma$-Banach algebra $V$ is called 2-torsion free if $2 x=0$ implies $x=0$, for all $x \in V$.

A $\Gamma$-ideal $\mathrm{I}$ of a $\Gamma$-Banach algebra $\mathrm{V}$ is said to be prime $\Gamma$-ideal if for any two $\Gamma$-ideals $\mathrm{A}$ and $\mathrm{B}$, $A \Gamma B \subseteq P \Rightarrow A \subseteq P$ or $B \subseteq P$

A $\Gamma$ - Banach algebra $\mathrm{V}$ is said to have a left (right) strong unity if there exists some $d \in V, \delta \in \Gamma$ such that $d \delta x=x(x \delta d=x), \forall x \in V$.

The Projective tensor norm $\|\cdot\|_{\gamma} \quad$ on $\mathrm{X} \otimes \mathrm{Y}$ is defined as $\|\mathrm{u}\|=\inf \left\{\sum_{\mathrm{i}}\left\|\mathrm{x}_{\mathrm{i}}\right\|\left\|\mathrm{y}_{\mathrm{i}}\right\|: u=\sum_{i} x_{i} \otimes y_{i}\right.$;

$x_{i} \in X, y_{i} \in Y$ \}, where the infimum is taken over all (finite) representations of $\mathrm{u}$. The completion of $(\mathrm{X} \otimes \mathrm{Y}$, $\left.\|\cdot\|_{\gamma}\right)$ is called the projective tensor product of $\mathrm{X}$ and $\mathrm{Y}$ and is denoted by $\mathrm{X} \otimes_{\gamma} \mathrm{Y}$.

Let $V$ and $V^{\prime}$ be $\Gamma$-Banach algebras over the fields $\mathrm{F}_{1}$ and $\mathrm{F}_{2}$ respectively isomorphic to which are a field

F. The projective tensor product $V \otimes_{p} V^{\prime}$ (with the projective tensor norm), is a $\Gamma \otimes \Gamma$-Banach algebra over $\mathrm{F}$, where multiplication is defined by the formula:

$(\mathrm{x} \otimes \mathrm{y})(\alpha \otimes \beta)\left(\mathrm{x}^{\prime} \otimes \mathrm{y}^{\prime}\right)=\left(\mathrm{x} \alpha \mathrm{x}^{\prime}\right) \otimes\left(\mathrm{y} \beta \mathrm{y}^{\prime}\right)$, where $x, y \in V ; \mathrm{x}^{\prime}, \mathrm{y}^{\prime} \in V^{\prime} ; \alpha, \beta \in \Gamma$.

An additive operator $\mathrm{d}$ on the $\Gamma$-Banach algebra $\mathrm{V}$ over a field $\mathrm{F}$ into itself is called a k-derivation if $\mathrm{d}(\mathrm{x} \gamma \mathrm{y})=\mathrm{d}(\mathrm{x}) \gamma \mathrm{y}+\mathrm{xk}(\gamma) \mathrm{y}+\mathrm{x} \gamma \mathrm{d}(\mathrm{y})$, for all $\mathrm{x}, \mathrm{y} \in \mathrm{V} ; \gamma \in \Gamma$, where $\mathrm{k}: \Gamma \rightarrow \Gamma$ is also a additive map. If $\mathrm{d}(\mathrm{x} \gamma \mathrm{x})=\mathrm{d}(\mathrm{x}) \gamma \mathrm{x}+\mathrm{x}$ $\mathrm{k}(\gamma) \mathrm{x}+\mathrm{x} \gamma \mathrm{d}(\mathrm{x})$ holds for all $\mathrm{x} \in \mathrm{V}$ and $\gamma \in \Gamma$, then $\mathrm{d}$ is called a Jordan $\mathrm{k}$-derivation on $\mathrm{V}$.

Let a and $\gamma$ be nonzero elements of $\mathrm{V}$ and $\Gamma$ respectively. The $\mathrm{d}: \mathrm{V} \rightarrow \mathrm{V}$ defined by $\mathrm{d}(\mathrm{x})=[\mathrm{a}, \mathrm{x}]_{\gamma}$ and $\mathrm{k}: \Gamma \rightarrow \Gamma$ defined by $\mathrm{k}(\beta)=[\gamma, \beta]_{\mathrm{a}}$ are two additive maps and $\mathrm{d}$ is a k-derivation on $\mathrm{V}$. Then we call $\mathrm{d}$ is an inner $\mathrm{k}$ derivation on $\mathrm{V}$.

Let $\mathrm{V}$ be a $\Gamma$-Banach algebra. A mapping $\mathrm{D}(.,):. \mathrm{V} \times \mathrm{V} \rightarrow \mathrm{V}$ is said to be symmetric bi-additive if it is additive in both arguments and $\mathrm{D}(\mathrm{x}, \mathrm{y})=\mathrm{D}(\mathrm{y}, \mathrm{x})$, for all $\mathrm{x}, \mathrm{y} \in \mathrm{V}$. By the trace of $\mathrm{D}(. .$,$) , we mean a map \mathrm{d}: \mathrm{V} \rightarrow \mathrm{V}$ defined by $\mathrm{d}(\mathrm{x})=\mathrm{D}(\mathrm{x}, \mathrm{x}), \forall \mathrm{x} \in \mathrm{V}$. A symmetric bi-additive map is called a symmetric bi-k-derivation if (a) $\mathrm{D}(\mathrm{x} \gamma \mathrm{y}$, $\mathrm{z})=\mathrm{D}(\mathrm{x}, \mathrm{z}) \mathrm{k}(\gamma) \mathrm{y}+\mathrm{x} \mathrm{k}(\gamma) \mathrm{D}(\mathrm{y}, \mathrm{x})(\mathrm{b}) \mathrm{D}(\mathrm{x}, \mathrm{y} \gamma \mathrm{z})=\mathrm{D}(\mathrm{x}, \mathrm{y}) \mathrm{k}(\gamma) \mathrm{z}+\mathrm{yk}(\gamma) \mathrm{D}(\mathrm{x}, \mathrm{z})$, for all $\mathrm{x}, \mathrm{y}, \mathrm{z} \in \mathrm{V} ; \gamma \in \Gamma$ and $\mathrm{k}: \Gamma \rightarrow \Gamma$ is a additive map. Since a map $\mathrm{D}(. .$,$) is symmetric bi-additive, the trace of \mathrm{D}(. .$,$) satisfies the relation$ $d(x+y)=d(x)+d(y)+2 D(x, y)$, for all $x, y \in V$ and is an even function.

Let $\mathrm{V}$ be a prime $\Gamma$ - Banach algebra such that $\mathrm{V} \Gamma \mathrm{V} \neq \mathrm{V}$. Denote $£=\{(\mathrm{I}, \mathrm{f}): \mathrm{I}(\neq 0)$ is an ideal of $\mathrm{V}$ and $\mathrm{f}$ : $\mathrm{I} \rightarrow \mathrm{V}$ is a $\Gamma$ - Banach algebra homomorphism $\}$ 
Define a relation $\sim$ on $£$ by $(\mathrm{I}, \mathrm{f}) \sim(\mathrm{J}, \mathrm{g}) \Leftrightarrow \exists \mathrm{K}(\neq 0) \subset \mathrm{I} \cap \mathrm{J}$ such that $\mathrm{f}=\mathrm{g}$ on $\mathrm{K}$. Since $\mathrm{V}$ is a prime $\Gamma$-Banach algebra, it is possible to find such a $\mathrm{K}$ and so " $\sim$ " is an equivalence relation on $£$. This gives a chance for us to get a partition of $£$. We then denote the equivalence class by $C l(1, f)=\hat{f}=\{g: J \rightarrow V \mid(1, f) \sim(J, g)\}$ and denote by $\mathrm{Q}$, the set of all equivalence Classes. Now we define an addition "+" and as follows

$\hat{f}+\hat{g}=C l(I, f)+C l(J, g)=C l(I \cap J, f+g)$.It can be easily shown that the addition "+" is well defined and $(\mathrm{Q},+)$ is an abelian group.

Since $\mathrm{V} \Gamma \mathrm{V} \neq \mathrm{V}$ and since $\mathrm{V}$ is a prime $\Gamma$ - Banach algebra $\mathrm{V} \Gamma \mathrm{V}(\neq 0)$ is an ideal of $\mathrm{V}$. We can take the homomorphism $1_{V \Gamma}: V \Gamma V \rightarrow V$ as a unit $\Gamma$ - Banach algebra homomorphism. Note that $V \beta V \neq 0$, for all $0 \neq \beta \in \Gamma$. So that $1_{V \beta}: V \beta V \rightarrow V$ is a nonzero $\Gamma$ - Banach algebra homomorphism. Define $\xi=\left\{\left(V \beta V, 1_{V \beta}\right) \mid 0 \neq \beta \in \Gamma\right\}$ and define a relation “ $\approx$ " on $\xi$ by $\left(\mathrm{V} \beta \mathrm{V}, 1_{V \beta}\right) \approx\left(\mathrm{V} \gamma \mathrm{V}, 1_{V \gamma}\right) \Leftrightarrow$ $\exists \mathrm{W}=\mathrm{V} \alpha \mathrm{V}(\neq 0) \subset \mathrm{V} \beta \mathrm{V} \cap \mathrm{V} \gamma \mathrm{V}$ such that $1_{V \beta}=1_{V \gamma}$ on $\mathrm{W}$. we can easily check that " $\approx$ " is an equivalence relation on $\xi$. Denote by $\mathrm{Cl}\left(\mathrm{V} \beta \mathrm{V}, 1_{V \beta}\right)=\hat{\beta}=\left\{\left(\mathrm{V} \gamma \mathrm{V}, 1_{V \gamma}\right):\left(\mathrm{V} \beta \mathrm{V}, 1_{V \beta}\right) \approx\left(\mathrm{V} \gamma \mathrm{V}, 1_{V \gamma}\right)\right\}$ and $\hat{\Gamma}=\{\hat{\beta} \mid 0 \neq \beta \in \Gamma\}$. Define an addition "+" on $\hat{\Gamma}$ as follows:

$\hat{\beta}+\hat{\delta}=c l\left(V \beta V, 1_{V \beta}\right)+c l\left(V \delta V, 1_{V \delta}\right)$

$=c l\left(V \beta V \cap V \delta V, 1_{V \beta}+1_{V \delta}\right)$, for every $\beta(\neq 0), \delta(\neq 0) \in \Gamma$. Then is an abelian group. Now we define a mapping (-,-,-): $Q \times \hat{\Gamma} \times Q \rightarrow Q,(\hat{f}, \hat{\beta}, \hat{g}) \mapsto \hat{f} \hat{\beta} \hat{g}$, as follows:

$\hat{\mathrm{f}} \hat{\beta} \hat{\mathrm{g}}=\mathrm{cl}(\mathrm{I}, \mathrm{f}) \mathrm{cl}\left(\mathrm{V} \beta \mathrm{V}, 1_{\mathrm{V} \beta}\right) \mathrm{cl}(\mathrm{J}, \mathrm{g})$

$=\mathrm{cl}\left(\mathrm{I} \Gamma \mathrm{V} \beta \mathrm{V} \Gamma \mathrm{J}, f I_{V \beta} g\right)$

Where IГV $\beta \mathrm{V} Г \mathrm{~J}=\left\{\sum_{i} u_{i} \alpha_{i} m_{i} \beta n_{i} \beta_{i} v_{i}: u_{i} \in I, v_{i} \in J ; m_{i}, n_{i} \in V ; \alpha_{i} \beta_{i} \in \Gamma\right\}$ is an ideal of $\mathrm{V}$ and $f 1_{V \beta} \mathrm{g}: \quad$ IГV $\beta \mathrm{V} \Gamma \mathrm{J} \rightarrow \mathrm{V}$ is a $\Gamma$ - Banach algebra homomorphism which is define as $f 1_{V \beta} \mathrm{g}$ $\left(\sum u_{i} \alpha_{i} m_{i} \beta n_{i} \beta_{i} v_{i}\right)=\sum_{i} f\left(u_{i}\right) \alpha_{i} 1_{V \beta}\left(m_{i} \beta n_{i}\right) \beta_{i} g\left(v_{i}\right)$ is a $\Gamma$ - Banach algebra homomorphism. Then for $\hat{f}, \hat{g}, \hat{h} \in Q ; \hat{\beta}, \hat{\gamma} \in \hat{\Gamma}$, we have

$$
\begin{gathered}
(\hat{f}+\hat{g}) \hat{\beta} \hat{h}=\hat{f} \hat{\beta} \hat{h}+\hat{g} \hat{\beta} \hat{h}, \hat{f}(\hat{\beta}+\hat{\gamma}) \hat{g}=\hat{f} \hat{\beta} \hat{g}+\hat{f} \hat{\gamma} \hat{g}, \hat{f} \hat{\beta}(\hat{g}+\hat{h})=\hat{f} \hat{\beta} \hat{g}+\hat{f} \hat{\beta} \hat{h} \\
(\hat{f} \hat{\beta} \hat{g}) \hat{\gamma} \hat{h}=\hat{f}(\hat{\beta} \hat{g} \hat{\gamma}) \hat{h}=\hat{f} \hat{\beta}(\hat{g} \hat{\gamma} \hat{h}), \hat{f} \hat{\beta} \hat{g}=\hat{0}, \forall \hat{f}, \hat{g}, \in Q \text { implies } \hat{\beta}=\hat{0} .
\end{gathered}
$$

Hence $\mathrm{Q}$ is a $\Gamma$-ring. Now we define scalar multiplication as $a \hat{f}=\operatorname{acl}(U, \hat{f})=c l(U, a f)$, $a \in F ; \hat{f} \in Q$. Then for $\hat{f}, \hat{g} \in Q ; a, b \in F$

$$
a(\hat{f}+\hat{g})=a \hat{f}+a \hat{g},(a+b) \hat{f}=a \hat{f}+a \hat{g},(a b) \hat{f}=a(b \hat{f}), 1 \cdot \hat{f}=\hat{f}
$$

Hence $Q(F)$ is a vector space. Now for $\hat{f}, \hat{g} \in Q ; \hat{\beta} \in \hat{\Gamma} ; a \in F$ we can show that $a(\hat{f} \hat{\beta} \hat{g})=(a \hat{f}) \hat{\beta} \hat{g}=\hat{f} \hat{\beta}(a \hat{g})$

Next define a norm on Q by $\|\hat{f}\|=\|(U, f)\|=\sup \{\|f(x)\|: x \in U,\|x\| \leq 1\}$

Then we find that $(\|\|, Q$.$) is a norm linear space. If \left\{\hat{f}_{n}\right\}$ is a Cauchy sequence in $\mathrm{Q}$, then for given $\epsilon<0, \exists$ positive integer $_{n_{0}}$ such that $m, n \geq n_{0} \Rightarrow\left\|\hat{f}_{n}-\hat{f}_{m}\right\|_{<\epsilon}$

$$
\begin{aligned}
& \Rightarrow \sup \left\{\left\|\hat{f}_{n}(x)-\hat{f}_{m}(x)\right\|: x \in U_{n} \cap U_{m} \text { and }\|x\| \leq 1\right\}_{<\epsilon} \\
& \Rightarrow\left\|f_{n}(x)-f_{m}(x)\right\|_{<\epsilon}, \quad x \in U_{n} \cap U_{m} \text { and }\|x\| \leq 1
\end{aligned}
$$




$$
\Rightarrow\left\{f_{n}(x)\right\} \text { is a Cauchy sequence in } \mathrm{M} \text {. }
$$

$$
\Rightarrow \exists\left(U_{0}, f_{0}\right) \in £ \text { such that } f_{n}(x) \rightarrow f_{0}(x) \text {, because the norm in Q is uniformly }
$$

continuous. So we can prove easily that $\hat{f}_{n} \rightarrow \hat{f}_{0} \in Q$. Therefore $\mathrm{Q}$ is a Banach Algebra over $\mathrm{F}$.

Moreover, for $\hat{f}, \hat{g} \in Q ; \hat{\beta} \in \hat{\Gamma}$, we have

$\|\hat{f}\| \hat{\beta}\|\hat{g}\|=\|\operatorname{cl}(I, f)\|\left\|\operatorname{cl}\left(V \beta V, 1_{V \beta}\right)\right\|\|\operatorname{cl}(J, g)\|$

$=\left\|\operatorname{cl}\left(\Pi \Gamma V \beta V \Gamma J, f 1_{V \beta} g\right)\right\|$

$=\sup \{\|f(u)\|: u \in I,\|u\| \leq 1\} \cdot \sup \left\{\left\|1_{V \beta}(x \beta y)\right\|: x \beta y \in V \beta V,\|x \beta y\| \leq 1\right\} \sup \{\|v\|: v \in J,\|v\| \leq 1\}$

$=\sup \left\{\|f(u)\| 1_{V \beta}(x \beta y)\|\| g(v)\|: u \in I, x \beta y \in V \beta V, v \in J ;\| u\|\leq 1\|, x \beta y\|\leq 1\|, v \| \leq 1\right\}$

$\geq \sup \left\{\|f(u)\| \mid\|\gamma\| 1_{v \beta}(x \beta y)\|\| \gamma^{\prime}\|\| g(v)\|: u \in I, x \beta y \in V \beta V, v \in J ;\| u\|\leq 1\|, x \beta y\|\leq 1\| v,\|\leq 1,\| \gamma\|\leq 1,\| \gamma^{\prime} \| \leq 1\right\}$

$=\sup \left\{\left\|f(u) \gamma 1_{V \beta}(x \beta y) \gamma^{\prime} g(v)\right\|: u \in I, x \beta y \in V \beta V, v \in J ;\|u\| \leq 1,\|x \beta y\| \leq 1,\|v\| \leq 1,\|\gamma\| \leq 1,\left\|\gamma^{\prime}\right\| \leq 1\right\}$

$=\sup \left\{\mid f 1_{V \beta} g\left(u>x \beta y \gamma^{\prime} v\right)\|: u \in I, x \beta y \in V \beta V, v \in J ;\| u \gamma x \beta y \gamma^{\prime} v \| \leq 1\right\}$

$=\left\|\operatorname{cl}\left(I \Gamma V \beta V \Gamma J, f 1_{V \beta} g\right)\right\|$

$=\left\|\operatorname{cl}(I, f) \operatorname{cl}\left(V \beta V, 1_{V \beta}\right) \operatorname{cl}(J, g)\right\|$

$=\|\hat{f} \hat{\beta} \hat{g}\|$

Thus $\mathrm{Q}$ is a $\hat{\Gamma}$-Banach algebra over F. Noticing that the mapping $\eta(\beta)=\hat{\beta}$ for every $0 \neq \beta \in \Gamma$ is an isomorphism. Therefore $\hat{\Gamma}$-Banach algebra $\mathrm{Q}$ is a $\Gamma$-Banach algebra.

The set $C_{\Gamma}=\{g \in Q \mid g \gamma f=f \gamma g, \forall f \in Q a n d \gamma \in \Gamma\}$, is called the extended centroid of $\Gamma$-Banach algebra $\mathrm{V}$ over $\mathrm{F}$. If $\mathrm{a} \gamma \mathrm{x} \beta \mathrm{b}=\mathrm{b} \gamma \mathrm{x} \beta \mathrm{a}$, for all $\mathrm{x} \in \mathrm{V}$ and $\beta, \gamma \in \Gamma$, where $\mathrm{a}(\neq 0), \mathrm{b} \in \mathrm{V}$ are fixed, then there exists $\lambda \in \mathrm{C}_{\Gamma}$ such that $b=\lambda \alpha a$ for $\alpha \in \Gamma$.

\section{The main results:}

Theorem3.1. If $\mathrm{d}_{1}$ and $\mathrm{d}_{2}$ be bounded $\mathrm{k}$ - and h-derivations on $\Gamma$-Banach algebras $V\left(F_{1}\right)$ and $V^{\prime}\left(F_{2}\right)$ respectively then there exists a bounded $\mathrm{h} \otimes \mathrm{k}$-derivation $\mathrm{d}$ on the projective tensor product $V \otimes_{p} V^{\prime}$ defined by the relation $\mathrm{d}(\mathrm{u})=\sum_{\mathrm{i}}\left[\mathrm{d}_{1}\left(\mathrm{x}_{\mathrm{i}}\right) \otimes \mathrm{y}_{\mathrm{i}}+\mathrm{x}_{\mathrm{i}} \otimes \mathrm{d}_{2}\left(\mathrm{y}_{\mathrm{i}}\right)\right]$, where $u=\sum_{i} x_{i} \otimes y_{i} \in V \otimes{ }_{p} V$.

Proof: $\quad$ Since $\quad d: V\left(F_{1}\right) \otimes_{p} V^{\prime}\left(F_{2}\right) \rightarrow V\left(F_{1}\right) \otimes_{p} V^{\prime}\left(F_{2}\right) \quad$ is $\quad$ define $\quad$ as $\mathrm{d}(\mathrm{u})=\sum_{\mathrm{i}}\left[\mathrm{d}_{1}\left(\mathrm{x}_{\mathrm{i}}\right) \otimes \mathrm{y}_{\mathrm{i}}+\mathrm{x}_{\mathrm{i}} \otimes \mathrm{d}_{2}\left(\mathrm{y}_{\mathrm{i}}\right)\right]$, where $u=\sum_{i} x_{i} \otimes y_{i} \in V\left(F_{1}\right) \otimes{ }_{p} V^{\prime}\left(F_{2}\right)$. Clearly d is well defined. For any arbitrary element $u=\sum_{i} x_{i} \otimes y_{i} \in V\left(F_{1}\right) \otimes_{p} V^{\prime}\left(F_{2}\right)$ and $\in>0$ we have $\|\mathrm{u}\|_{\mathrm{p}}+\in \geq \sum_{\mathrm{i}=1}^{\mathrm{n}}\left\|\mathrm{x}_{\mathrm{i}}\right\|\left\|\mathrm{y}_{\mathrm{i}}\right\|$, from the definition of projective norm. Now $\|\mathrm{d}(\mathrm{u})\|_{\mathrm{p}}=\left\|\sum_{\mathrm{i}}\left[\mathrm{d}_{1}\left(\mathrm{x}_{\mathrm{i}}\right) \otimes \mathrm{y}_{\mathrm{i}}+\mathrm{x}_{\mathrm{i}} \otimes \mathrm{d}_{2}\left(\mathrm{y}_{\mathrm{i}}\right)\right]\right\|_{\mathrm{p}}$. Thus $\leq \sum_{i}\left\lfloor\left\|d_{1}\left(x_{i}\right) \otimes y_{i}\right\|+\left\|x_{i} \otimes d_{2}\left(y_{i}\right)\right\|\right\rfloor$ 
$=\sum_{i}\left[\left\|d_{1}\left(x_{i}\right)\right\|\left\|y_{i}\right\|+\left\|x_{i}\right\|\left\|d_{2}\left(y_{i}\right)\right\|\right]$

$\leq\left(\left\|\mathrm{d}_{1}\right\|+\left\|\mathrm{d}_{2}\right\|\right) \sum_{\mathrm{i}}\left\|\mathrm{x}_{\mathrm{i}}\right\|\left\|\mathrm{y}_{\mathrm{i}}\right\|$

$\leq \mathrm{k}\left(\|\mathrm{u}\|_{\mathrm{p}}+\in\right)$, where $\mathrm{k}=\left\|\mathrm{d}_{1}\right\|+\left\|\mathrm{d}_{2}\right\|$

Thus $\|\mathrm{d}(\mathrm{u})\|_{\mathrm{p}} \leq \mathrm{k}\left(\|\mathrm{u}\|_{\mathrm{p}}+\in\right)$. Since the left hand side is independent of $\in$ and $\in$ was arbitrary, it follows that $\|\mathrm{d}(\mathrm{u})\|_{\mathrm{p}} \leq \mathrm{k}\|\mathrm{u}\|_{\mathrm{p}}$, for every $u \in V\left(F_{1}\right) \otimes_{p} V^{\prime}\left(F_{2}\right)$. Consequently $\mathrm{D}$ is bounded.

To show that $\mathrm{d}$ is a $\mathrm{k} \otimes \mathrm{h}$-derivation, we suppose that $\mathrm{u}=\mathrm{x} \otimes \mathrm{y}$ and $\mathrm{v}=\mathrm{x}^{\prime} \otimes \mathrm{y}^{\prime}$ are two elements of $V\left(F_{1}\right) \otimes_{p} V^{\prime}\left(F_{2}\right)$. Then

$d\left((x \otimes y)(\alpha \otimes \beta)\left(x^{\prime} \otimes y^{\prime}\right)\right)$, where $\alpha \otimes \beta \in \Gamma \otimes_{\mathrm{p}} \Gamma^{\prime}$

$=\mathrm{d}\left(\mathrm{x} \alpha \mathrm{x}^{\prime} \otimes \mathrm{y} \beta \mathrm{y}^{\prime}\right)$

$=\mathrm{d}_{1}\left(\mathrm{x} \alpha \mathrm{x}^{\prime}\right) \otimes \mathrm{y} \beta \mathrm{y}^{\prime}+\mathrm{x} \alpha \mathrm{x}^{\prime} \otimes \mathrm{d}_{2}\left(\mathrm{y} \beta \mathrm{y}^{\prime}\right)$

$=\left[d_{1}(x) \alpha x^{\prime}+x k(\alpha) x^{\prime}+x \alpha d_{1}\left(x^{\prime}\right)\right] \otimes \mathrm{y}^{\prime} \mathrm{y}^{\prime}+\mathrm{x} \alpha \mathrm{x}^{\prime} \otimes\left[d_{2}(y) \beta y^{\prime}+y h(\beta) y^{\prime}+y \beta d_{2}\left(y^{\prime}\right)\right]$

$=\mathrm{d}(\mathrm{x} \otimes \mathrm{y})(\alpha \otimes \beta)\left(\mathrm{x}^{\prime} \otimes \mathrm{y}^{\prime}\right)+(\mathrm{x} \otimes \mathrm{y})(\mathrm{k} \otimes \mathrm{h})(\alpha \otimes \beta)\left(\mathrm{x}^{\prime} \otimes \mathrm{y}^{\prime}\right)+(\mathrm{x} \otimes \mathrm{y})(\alpha \otimes \beta) \mathrm{d}\left(\mathrm{x}^{\prime} \otimes \mathrm{y}^{\prime}\right)$,

where $(\mathrm{k} \otimes \mathrm{h})(\alpha \otimes \beta)=\mathrm{k}(\alpha) \otimes \beta+\alpha \otimes \mathrm{h}(\beta)$

Similarly, if $\mathrm{u}=\sum_{\mathrm{i}} \mathrm{x}_{\mathrm{i}} \otimes \mathrm{y}_{\mathrm{i}}$ and $\mathrm{v}=\sum_{\mathrm{j}} \mathrm{x}_{\mathrm{j}}^{\prime} \otimes \mathrm{y}_{\mathrm{j}}^{\prime}$ be two element of $V\left(F_{1}\right) \otimes_{p} V^{\prime}\left(F_{2}\right)$ then summing over $i$ and $j$ we can prove easily that $\mathrm{d}(\mathrm{u}(\alpha \otimes \beta) \mathrm{v})=\mathrm{d}(\mathrm{u})(\alpha \otimes \beta) \mathrm{v}+\mathrm{u}[(\mathrm{k} \otimes \mathrm{h})(\alpha \otimes \beta)] \mathrm{v}+\mathrm{u}(\alpha \otimes \beta) \mathrm{d}(\mathrm{v})$

So, $\mathrm{d}$ is a $\mathrm{k} \otimes \mathrm{h}$-derivation.

Theorem3.2. Let $V(F)$ and $V^{\prime}\left(F^{\prime}\right)$ be two $\Gamma$-Banach algebra and $\Gamma^{\prime}$-Banach algebra respectively with e $\delta \mathrm{x}$ $=\mathrm{x} \delta \mathrm{e}=\mathrm{x}, \forall \mathrm{x} \in \mathrm{V}, \mathrm{e} \in \mathrm{V}, \delta \in \Gamma$ and $e^{\prime} \delta^{\prime} y=y \delta^{\prime} e^{\prime}=y, \forall y \in V^{\prime}, e^{\prime} \in V^{\prime}, \delta^{\prime} \in \Gamma^{\prime}$. If $\mathrm{d}_{1}$ and $\mathrm{d}_{2}$ are k-and hinner derivation on $V(F)$ and $V^{\prime}\left(F^{\prime}\right)$ respectively implemented by $(\mathrm{a}, \delta)$ and $\left(\mathrm{b}, \delta^{\prime}\right)$ respectively then $\mathrm{d}$ is a $\mathrm{k} \otimes \mathrm{h}$ - inner deviation on $V \otimes_{p} V^{\prime}$ implemented by $\left(\mathrm{a} \otimes \mathrm{e}^{\prime}+\mathrm{e} \otimes \mathrm{b}, \delta \otimes \delta^{\prime}\right)$.

Proof: Let $\mathrm{d}_{1}$ and $\mathrm{d}_{2}$ be k- and h-inner derivations on $V(F)$ and $V^{\prime}\left(F^{\prime}\right)$ respectively implemented by $(\mathrm{a}, \delta)$ and $\left(b, \delta^{\prime}\right)$ i.e.

$\mathrm{d}_{1}(\mathrm{x})=[\mathrm{a}, \mathrm{x}]_{\delta}$, where $\mathrm{k}(\alpha)=[\delta, \alpha]_{\mathrm{a}}$

$\mathrm{d}_{2}(\mathrm{y})=[\mathrm{b}, \mathrm{y}]_{\delta^{\prime}}$, where $h(\beta)=\left[\delta^{\prime}, \beta\right]_{b}$

Now $\mathrm{d}(\mathrm{u})=\mathrm{d}\left(\sum_{\mathrm{i}} \mathrm{x}_{\mathrm{i}} \otimes \mathrm{y}_{\mathrm{i}}\right)$

$$
\begin{aligned}
& =\sum_{i}\left[d_{1}\left(x_{i}\right) \otimes y_{i}+x_{i} \otimes d_{2}\left(y_{i}\right)\right] \\
& =\sum_{\mathrm{i}}\left[\left[\mathrm{a}, \mathrm{x}_{\mathrm{i}}\right]_{\delta} \otimes \mathrm{y}_{\mathrm{i}}+\mathrm{x}_{\mathrm{i}} \otimes\left[\mathrm{b}, \mathrm{y}_{\mathrm{i}}\right]_{\delta^{\prime}}\right] \\
& =\sum_{\mathrm{i}}\left[\left(\mathrm{a} \delta \mathrm{x}_{\mathrm{i}}-\mathrm{x}_{\mathrm{i}} \delta \mathrm{a}\right) \otimes \mathrm{y}_{\mathrm{i}}+\mathrm{x}_{\mathrm{i}} \otimes\left(\mathrm{b}^{\prime} \mathrm{y}_{\mathrm{i}}-\mathrm{y}_{\mathrm{i}} \delta^{\prime} \mathrm{b}\right)\right] \\
& =\sum_{\mathrm{i}}\left[\mathrm{a} \delta \mathrm{x}_{\mathrm{i}} \otimes \mathrm{y}_{\mathrm{i}}-\mathrm{x}_{\mathrm{i}} \delta \mathrm{a} \otimes \mathrm{y}_{\mathrm{i}}+\mathrm{x}_{\mathrm{i}} \otimes \mathrm{b}^{\prime} \mathrm{y}_{\mathrm{i}}-\mathrm{x}_{\mathrm{i}} \otimes \mathrm{y}_{\mathrm{i}} \delta^{\prime} \mathrm{b}\right]
\end{aligned}
$$




$$
\begin{aligned}
&=\sum_{\mathrm{i}}\left[\mathrm{a} \delta \mathrm{x}_{\mathrm{i}} \otimes \mathrm{e}^{\prime} \delta^{\prime} \mathrm{y}_{\mathrm{i}}-\mathrm{x}_{\mathrm{i}} \delta \mathrm{a} \otimes \mathrm{y}^{\prime} \mathrm{e}^{\prime}+\mathrm{e} \delta \mathrm{x}_{\mathrm{i}} \otimes \mathrm{b}^{\prime} \mathrm{y}_{\mathrm{i}}-\mathrm{x}_{\mathrm{i}} \delta \mathrm{e} \otimes \mathrm{y}_{\mathrm{i}} \delta^{\prime} \mathrm{b}\right] \\
&= \sum_{i}\left[\left(a \otimes e^{\prime}\right)\left(\delta \otimes \delta^{\prime}\right)\left(x_{i} \otimes y_{i}\right)-\left(x_{i} \otimes y_{i}\right)\left(\delta \otimes \delta^{\prime}\right)\left(\mathrm{a} \otimes \mathrm{e}^{\prime}\right)+(\mathrm{e} \otimes \mathrm{b})\left(\delta \otimes \delta^{\prime}\right)\right. \\
&\left.\left(\mathrm{x}_{\mathrm{i}} \otimes \mathrm{y}_{\mathrm{i}}\right)-\left(\mathrm{x}_{\mathrm{i}} \otimes \mathrm{y}_{\mathrm{i}}\right)\left(\delta \otimes \delta^{\prime}\right)(\mathrm{e} \otimes \mathrm{b})\right] \\
&=\sum_{i}\left[\left(a \otimes e^{\prime}+e \otimes b\right)\left(\delta \otimes \delta^{\prime}\right)\left(x_{i} \otimes y_{i}\right)-\left(x_{i} \otimes y_{i}\right)\left(\delta \otimes \delta^{\prime}\right)\left(\mathrm{a} \otimes \mathrm{e}^{\prime}+\mathrm{e} \otimes \mathrm{b}\right)\right] \\
&=\sum_{i}\left[a \otimes e^{\prime}+e \otimes b, x_{i} \otimes y_{i}\right]_{\delta \otimes \delta^{\prime}} \\
&=\sum_{i}\left[a \otimes e^{\prime}+e \otimes b, u\right]_{\delta \otimes \delta^{\prime}}
\end{aligned}
$$

Now, we define $\mathrm{k} \otimes \mathrm{h}: \Gamma \otimes \Gamma \rightarrow \Gamma \otimes \Gamma$ by

$(\mathrm{k} \otimes \mathrm{h})(\alpha \otimes \beta)=\left[\delta \otimes \delta^{\prime}, \alpha \otimes \beta\right]_{\mathrm{a} \otimes \mathrm{e}^{\prime}+\mathrm{e} \otimes \mathrm{b}}$

It can be easily prove that

$$
\begin{aligned}
& \mathrm{d}\left((\mathrm{x} \otimes \mathrm{y})(\alpha \otimes \beta)\left(\mathrm{x}^{\prime} \otimes \mathrm{y}^{\prime}\right)\right)=\mathrm{d}(\mathrm{x} \otimes \mathrm{y})(\alpha \otimes \beta)\left(\mathrm{x}^{\prime} \otimes \mathrm{y}^{\prime}\right)+(\mathrm{x} \otimes \mathrm{y})(\mathrm{k} \otimes \mathrm{h})(\alpha \otimes \beta) \\
& \left(\mathrm{x}^{\prime} \otimes \mathrm{y}^{\prime}\right)+(\mathrm{x} \otimes \mathrm{y})(\alpha \otimes \beta) \mathrm{d}\left(\mathrm{x}^{\prime} \otimes \mathrm{y}^{\prime}\right)
\end{aligned}
$$

Therefore $\mathrm{d}$ is a $\mathrm{k} \otimes \mathrm{h}$-inner derivation on $V \otimes_{p} V^{\prime}$ implemented by $\left(\mathrm{a} \otimes \mathrm{e}^{\prime}+\mathrm{e} \otimes \mathrm{b}, \delta \otimes \delta^{\prime}\right)$

Theorem3.3. If $\mathrm{d}_{1}$ and $\mathrm{d}_{2}$ are $\mathrm{k}$-and h-Jordan derivations, then $\mathrm{d}$ is an $\mathrm{h} \otimes \mathrm{k}$-Jordan derivation.

Proof: Obvious.

\section{Remarks:}

(i) The converse of the above three theorems are also true.

(ii) If $u=a \otimes e^{\prime} \in V(F) \otimes_{p} V^{\prime}\left(F^{\prime}\right)$, then from the definition of $\mathrm{d}$ in theorem 3.2, we get $\mathrm{d}(\mathrm{u})=\mathrm{d}_{1}(\mathrm{a}) \otimes \mathrm{e}^{\prime}$, because $\mathrm{d}_{2}\left(\mathrm{e}^{\prime}\right)=0$

(iii) If $u^{\prime}=e \otimes b \in V(F) \otimes_{p} V^{\prime}\left(F^{\prime}\right)$, then from the definition of $\mathrm{d}$ in theorem 3.2, we get $\mathrm{d}(\mathrm{u})=\mathrm{e} \otimes \mathrm{d}_{2}(\mathrm{~b})$, because $\mathrm{d}_{1}(\mathrm{e})=0$

Theorem3.4. If $\mathrm{d}_{1}, \mathrm{~d}_{2}$ and $\mathrm{d}$ are $\mathrm{k}$ - ,h- and $\mathrm{k} \otimes \mathrm{h}$-derivations respectively related as in theorem 3.1,3.2 and 3.3 , then

$$
\|\mathrm{d}\| \leq\left\|\mathrm{d}_{1}\right\|+\left\|\mathrm{d}_{2}\right\| \leq 2\|\mathrm{~d}\|
$$

Proof: we already proof in theorem 3.1 is that

$$
\|d\| \leq\left(\left\|d_{1}\right\|+\left\|d_{2}\right\|\right)(1+\in)
$$

Since $\in$ was arbitrary, it follows that

$$
\|\mathrm{d}\| \leq\left\|\mathrm{d}_{1}\right\|+\left\|\mathrm{d}_{2}\right\|
$$

Next let $\mathrm{x} \in \mathrm{V}$ such that $\|\mathrm{x}\|=1$. Then

$$
\begin{aligned}
& \left\|\frac{\mathrm{x}}{\mathrm{k}} \otimes \mathrm{e}^{\prime}\right\|=\left\|\frac{\mathrm{x}}{\mathrm{k}}\right\|\left\|\mathrm{e}^{\prime}\right\|=1 \text { where }\left\|\mathrm{e}^{\prime}\right\|=\mathrm{k} \neq 0 \\
& \text { Now }\|\mathrm{d}\|=\sup _{\mathrm{u}}\left\{\mathrm{d}(\mathrm{u})\left\|_{\mathrm{p}}:\right\| \mathrm{u} \|_{\mathrm{p}}=1\right\} \\
& \geq\left\|\mathrm{d}\left(\frac{\mathrm{x}}{\mathrm{k}} \otimes \mathrm{e}^{\prime}\right)\right\|_{\mathrm{p}} \\
& \quad=\left\|\mathrm{d}_{1}\left(\frac{\mathrm{x}}{\mathrm{k}}\right) \otimes \mathrm{e}^{\prime}\right\|_{\mathrm{p}}, \text { since } \mathrm{d}_{2}\left(\mathrm{e}^{\prime}\right)=0
\end{aligned}
$$




$$
=\left\|\mathrm{d}_{1}(\mathrm{x})\right\|
$$

Thus $\|d\| \geq\left\|d_{1}(x)\right\|$, for every $\mathrm{x} \in \mathrm{V}(\mathrm{F})$ with $\|\mathrm{x}\|=1$ and which implies $\|\mathrm{d}\| \geq\left\|\mathrm{d}_{1}\right\|$

Similarly, we can prove that $\|\mathrm{d}\| \geq\left\|\mathrm{d}_{2}\right\|$

$$
\therefore\left\|\mathrm{d}_{1}\right\|+\left\|\mathrm{d}_{2}\right\| \leq 2\|\mathrm{~d}\|
$$

The inequalities (1) and (2) together implies that $\|\mathrm{d}\| \leq\left\|\mathrm{d}_{1}\right\|+\left\|\mathrm{d}_{2}\right\| \leq 2\|\mathrm{~d}\|$.

Example1. Let $\mathrm{V}$ be the set of all $2 \times 2$ matrices of the type $\left(\begin{array}{cc}\mathrm{a} & \mathrm{b} \\ -\overline{\mathrm{b}} & \overline{\mathrm{a}}\end{array}\right)$ where $\mathrm{a}, \mathrm{b}$ are complex numbers and $\overline{\mathrm{a}}, \overline{\mathrm{b}}$ are their conjugates respectively and $\Gamma$ be the set of all $2 \times 2$ matrices of the type $\left(\begin{array}{ll}\mathrm{x} & 0 \\ 0 & \mathrm{x}\end{array}\right)$, where $\mathrm{x}$ is a real number. Then $\mathrm{V}$ be a $\Gamma$-Banach algebra over $\mathrm{F}=\mathrm{R}$ with respect to usual matrix addition and multiplication and the norm is defined by

$\left\|\left(\begin{array}{cc}\mathrm{a} & \mathrm{b} \\ -\overline{\mathrm{b}} & \overline{\mathrm{a}}\end{array}\right)\right\|=\max \{|\mathrm{a}|,|\mathrm{b}|\}$ and $\left\|\left(\begin{array}{cc}\mathrm{x} & 0 \\ 0 & \mathrm{x}\end{array}\right)\right\|=|\mathrm{x}|$.

Let $V^{\prime}$ be the set of all $2 \times 2$ matrix of the type $\left(\begin{array}{ll}\mathrm{x} & \mathrm{y} \\ \mathrm{u} & \mathrm{v}\end{array}\right)$, where $\mathrm{x}, \mathrm{y}, \mathrm{u}, \mathrm{v}$ are real numbers and $\Gamma^{\prime}=\Gamma$ and norm is defined as the norm is defined for $\mathrm{V}$ and $\Gamma$.Then $V^{\prime}\left(F^{\prime}\right)$ is a $\Gamma^{\prime}$ - Banach algebra, where $F^{\prime}=R$.

Let $d_{1}$ and $d_{2}$ are k- and h-derivations implemented by $(A, \delta)$ and $\left(B, \delta^{\prime}\right)$ respectively, where

$A=\left(\begin{array}{cc}3 i & 2 i \\ 2 i & -3 i\end{array}\right) \in V, B=\left(\begin{array}{cc}5 & 3 \\ 2 & 1 i\end{array}\right) \in V^{\prime}$

$\delta=\left(\begin{array}{cc}-1 & 0 \\ 0 & -1\end{array}\right) \in \Gamma, \delta^{\prime}=\left(\begin{array}{ll}2 & 0 \\ 0 & 2\end{array}\right) \in \Gamma^{\prime}$

Since $B_{1}=\left\{e_{1}=\left(\begin{array}{ll}1 & 0 \\ 0 & 1\end{array}\right), e_{2}=\left(\begin{array}{cc}i & 0 \\ 0 & -i\end{array}\right), e_{3}=\left(\begin{array}{cc}0 & -1 \\ 1 & 0\end{array}\right), e_{4}=\left(\begin{array}{ll}0 & i \\ i & 0\end{array}\right)\right\}$ is a basis for $\mathrm{V}(\mathrm{F})$.

Now $d_{1}\left(e_{1}\right)=\left[A, e_{1}\right]_{\delta}$

$$
\begin{aligned}
& =A \delta e_{1}-e_{1} \delta A \\
& =\left(\begin{array}{cc}
3 i & 2 i \\
2 i & -3 i
\end{array}\right)\left(\begin{array}{cc}
-1 & 0 \\
0 & -1
\end{array}\right)\left(\begin{array}{cc}
1 & 0 \\
0 & 1
\end{array}\right)-\left(\begin{array}{cc}
1 & 0 \\
0 & 1
\end{array}\right)\left(\begin{array}{cc}
-1 & 0 \\
0 & -1
\end{array}\right)\left(\begin{array}{cc}
3 i & 2 i \\
2 i & -3 i
\end{array}\right) \\
& =\left(\begin{array}{ll}
0 & 0 \\
0 & 0
\end{array}\right)
\end{aligned}
$$

Similarly, $d_{1}\left(e_{2}\right)=\left(\begin{array}{cc}0 & -4 \\ 4 & 0\end{array}\right), d_{1}\left(e_{3}\right)=\left(\begin{array}{cc}-4 i & 6 i \\ 6 i & 4 i\end{array}\right), d_{1}\left(e_{4}\right)=\left(\begin{array}{cc}0 & 6 \\ -6 & 0\end{array}\right)$.

Hence the matrix representation of $d_{1}$ with respect to $B_{1}$ is

$$
\left[d_{1}\right]_{B_{1}}=\left(\begin{array}{cccc}
0 & 0 & -4 i & 0 \\
0 & -4 & 6 i & 6 \\
0 & 4 & 6 i & -6 \\
0 & 0 & 4 i & 0
\end{array}\right)
$$


Therefore $\left\|\mathrm{d}_{1}\right\|=6$

Also $B_{2}=\left\{e_{1}^{\prime}=\left(\begin{array}{ll}1 & 0 \\ 0 & 0\end{array}\right), e_{2}^{\prime}=\left(\begin{array}{ll}0 & 1 \\ 0 & 0\end{array}\right), e_{3}^{\prime}=\left(\begin{array}{ll}0 & 0 \\ 1 & 0\end{array}\right), e_{4}^{\prime}=\left(\begin{array}{ll}0 & 0 \\ 0 & 1\end{array}\right)\right\}$ is a basis for $V^{\prime}$. Similarly,

with respect to this basis, we find $\left[d_{2}\right]_{B_{2}}=\left(\begin{array}{cccc}0 & -6 & 4 & 0 \\ -4 & 8 & 0 & 4 \\ 6 & 0 & -8 & -6 \\ 0 & 6 & -4 & 0\end{array}\right)$ and $\left\|d_{2}\right\|=8$.

Next we wish to find the matrix representation of $\mathrm{k} \otimes \mathrm{h}$-derivation d. Clearly $B_{3}=\left\{e_{i} \otimes e_{j}: i=1,2,3,4 ; j=1,2,3,4\right\}$ is a basis for $V(F) \otimes_{p} V^{\prime}\left(F^{\prime}\right)$.

$$
\begin{aligned}
d\left(e_{1} \otimes e_{1}{ }^{\prime}\right) & =d_{1}\left(e_{1}\right) \otimes e_{1}^{\prime}+e_{1} \otimes d_{2}\left(e_{1}^{\prime}\right) \\
& =\left(\begin{array}{ll}
0 & 0 \\
0 & 0
\end{array}\right) \otimes\left(\begin{array}{ll}
1 & 0 \\
0 & 0
\end{array}\right)+\left(\begin{array}{ll}
1 & 0 \\
0 & 1
\end{array}\right) \otimes\left(\begin{array}{cc}
0 & -6 \\
4 & 0
\end{array}\right) \\
& =\left(\begin{array}{llll}
0 & 0 & 0 & 0 \\
0 & 0 & 0 & 0 \\
0 & 0 & 0 & 0 \\
0 & 0 & 0 & 0
\end{array}\right)+\left(\begin{array}{cccc}
0 & 0 & -6 & 0 \\
4 & 0 & 0 & 0 \\
0 & 0 & 0 & -6 \\
0 & 4 & 0 & 0
\end{array}\right) \\
& =\left(\begin{array}{cccc}
0 & 0 & -6 & 0 \\
4 & 0 & 0 & 0 \\
0 & 0 & 0 & -6 \\
0 & -4 & 0 & 0
\end{array}\right)
\end{aligned}
$$

Similarly,

$$
\begin{aligned}
& d\left(e_{4} \otimes e_{2}^{\prime}\right)=\left(\begin{array}{cccc}
0 & -4 i & 0 & 6+8 i \\
0 & 0 & 0 & 4 i \\
-4 i & 0 & -6+8 i & 0 \\
0 & 0 & 4 i & 0
\end{array}\right), d\left(e_{4} \otimes e_{3}^{\prime}\right)=\left(\begin{array}{cccc}
0 & 6 i & 0 & 0 \\
0 & 6-8 i & 0 & -6 i \\
6 i & 0 & 0 & 0 \\
-6-8 i & 0 & -6 i & 0
\end{array}\right), d\left(e_{4} \otimes e_{4}^{\prime}\right)=\left(\begin{array}{cccc}
0 & 0 & 0 & 6 i \\
0 & -4 i & 0 & 6 \\
0 & 0 & 6 i & 0 \\
-4 i & 0 & -6 & 0
\end{array}\right) \\
& d\left(e_{1} \otimes e_{2}^{\prime}\right)=\left(\begin{array}{cccc}
-4 & 0 & 8 & 0 \\
0 & 0 & 4 & 0 \\
0 & -4 & 0 & 8 \\
0 & 0 & 0 & 4
\end{array}\right), d\left(e_{1} \otimes e_{3}^{\prime}\right)=\left(\begin{array}{cccc}
6 & 0 & 0 & 0 \\
-8 & 0 & -6 & 0 \\
0 & 6 & 0 & 0 \\
0 & -8 & 0 & -6
\end{array}\right), d\left(e_{1} \otimes e_{4}^{\prime}\right)=\left(\begin{array}{cccc}
0 & 0 & 6 & 0 \\
-4 & 0 & 0 & 0 \\
0 & 0 & 0 & 6 \\
0 & -4 & 0 & 0
\end{array}\right) \\
& d\left(e_{2} \otimes e_{1}^{\prime}\right)=\left(\begin{array}{cccc}
0 & -4 & -6 i & 0 \\
4 i & 0 & 0 & 0 \\
4 & 0 & 0 & 6 i \\
0 & -4 i & 0 & 0
\end{array}\right), d\left(e_{2} \otimes e_{2}^{\prime}\right)=\left(\begin{array}{cccc}
-4 i & 0 & 8 i & -4 \\
0 & 0 & 4 i & 0 \\
0 & 4 i & 4 & 0 \\
0 & 0 & 0 & -4 i
\end{array}\right), d\left(e_{2} \otimes e_{3}^{\prime}\right)=\left(\begin{array}{cccc}
6 i & 0 & 0 & 0 \\
-8 i & -4 & -6 i & 0 \\
0 & -6 i & 0 & 0 \\
4 & 8 i & 0 & 6 i
\end{array}\right)
\end{aligned}
$$


$d\left(e_{2} \otimes e_{4}^{\prime}\right)=\left(\begin{array}{cccc}0 & 0 & 6 i & 0 \\ -4 i & 0 & 0 & 0 \\ 0 & 0 & 0 & -6 i \\ 0 & 4 i & 4 & 0\end{array}\right), d\left(e_{3} \otimes e_{1}^{\prime}\right)=\left(\begin{array}{cccc}-4 i & 6 i & 0 & 6 \\ 0 & -4 & 0 & 0 \\ 6 i & 4 i & -6 & 0 \\ 4 & 0 & 4 & 4\end{array}\right), d\left(e_{3} \otimes e_{2}^{\prime}\right)=\left(\begin{array}{cccc}0 & 4 & -4 i & 6 i-8 \\ 0 & 0 & 0 & -4 \\ -4 & 0 & 8+6 i & 4 i \\ 0 & 0 & 4 & 0\end{array}\right)$

$d\left(e_{3} \otimes e_{3}^{\prime}\right)=\left(\begin{array}{cccc}0 & -6 & 0 & 0 \\ -4 i & 8+6 i & 0 & -6 \\ 6 & 0 & 0 & 0 \\ -8+6 i & 4 i & -6 & 0\end{array}\right), d\left(e_{3} \otimes e_{4}^{\prime}\right)=\left(\begin{array}{cccc}0 & 0 & 0 & -6 \\ 0 & 4 & -4 i & 6 i \\ 0 & 0 & 6 & 0 \\ -4 & 0 & 6 i & 4 i\end{array}\right), d\left(e_{4} \otimes e_{1}^{\prime}\right)=\left(\begin{array}{cccc}0 & 6 & 0 & -6 i \\ 0 & 4 i & 0 & 0 \\ -6 & 0 & -6 i & 0 \\ 4 i & 0 & 0 & 0\end{array}\right)$

The matrix representation of $\mathrm{d}$ with respect to the basis $B_{3}$ is

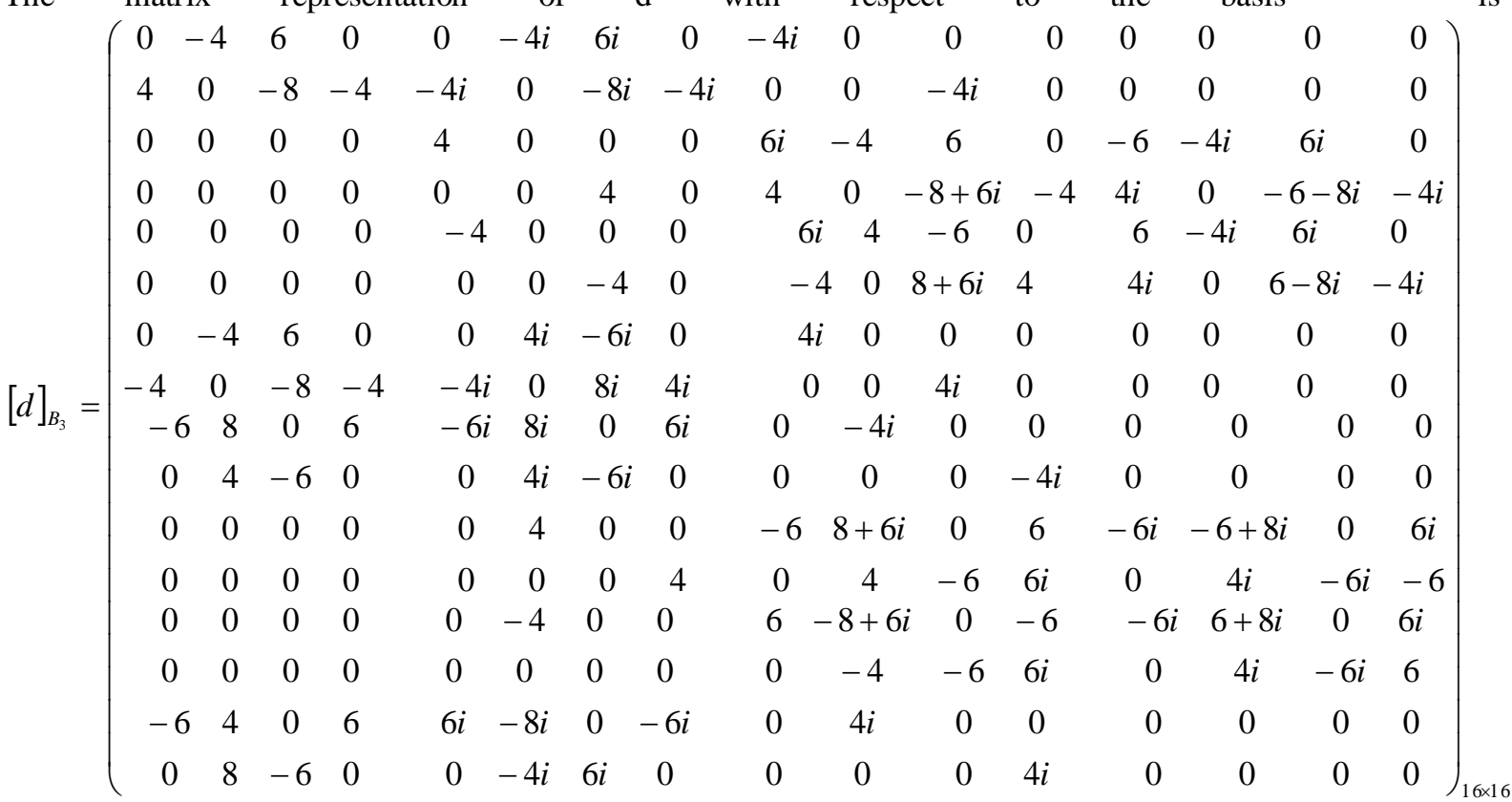

Therefore $\|\mathrm{d}\|=10$.Thus the strict inequality $\|\mathrm{d}\|<\left\|\mathrm{d}_{1}\right\|+\left\|\mathrm{d}_{2}\right\|<2\|\mathrm{~d}\|$ holds.

Lemma3.5 Let $\mathrm{V}$ be a 2-torsion free prime $\Gamma$-Banach algebra, $\mathrm{D}($...) the symmetric bi-k-derivation of $\mathrm{V}$ and $\mathrm{d}$ the trace of $\mathrm{D}(. .$.$) . If \mathrm{a} \gamma \mathrm{d}(\mathrm{x})=0$ for all $\mathrm{x} \in \mathrm{V}$ and $\gamma \in \Gamma$, where $\mathrm{a}$ is a fixed element of $\mathrm{V}$, then $\mathrm{a}=0$ or $\mathrm{D}=0$.

Lemma3.6 Let $\mathrm{V}$ be a 2-torsion free prime $\Gamma$-Banach algebra, $D_{1}(.,$.$) and \mathrm{D}_{2}(.,$.$) the symmetric bi-k-$ derivations on $\mathrm{V}$ and $\mathrm{d}_{1}$ and $\mathrm{d}_{2}$ the traces of $\mathrm{D}_{1}(.,$.$) and \mathrm{D}_{2}$ (...) respectively. If $\mathrm{d}_{1}(\mathrm{x}) \gamma \mathrm{d}_{2}(\mathrm{y})=\mathrm{d}_{2}(\mathrm{x}) \gamma$ $d_{1}$ (y) for all $x, y \in V$ and $\gamma \in \Gamma$ and $d_{1} \neq 0$, then there exists $\lambda \in C_{\Gamma}$ such that $d_{2}(x)=\lambda \alpha d_{1}(x)$ for $\alpha \in \Gamma$, where $\mathrm{C}_{\Gamma}$ is the extended centroid of $\mathrm{V}$.

Theorem3.7. Let $\mathrm{V}$ be a 2-torsion free prime $\Gamma$-Banach algebra, $\mathrm{D}_{1}(.,),. \mathrm{D}_{2}(. .$,$) and \mathrm{D}_{3}(.,$.$) and \mathrm{D}_{4}(.,$. the symmetric bi-k-derivations on $\mathrm{V}$ and $\mathrm{d}_{1}, \mathrm{~d}_{2}, \mathrm{~d}_{3}$ and $\mathrm{d}_{4}$ traces of $\mathrm{D}_{1}(.,),. \mathrm{D}_{2}(.,),. \mathrm{D}_{3}(. .$,$) and \mathrm{D}_{4}(.,$. respectively. If

$\mathrm{d}_{1}(\mathrm{x}) \gamma \mathrm{d}_{2}(\mathrm{y})=\mathrm{d}_{3}(\mathrm{x}) \gamma \mathrm{d}_{4}(\mathrm{y})$

for all $\mathrm{x}, \mathrm{y} \in \mathrm{V}$ and $\gamma \in \Gamma$ and $\mathrm{d}_{1} \neq 0 \neq \mathrm{d}_{4}$, then there exists $\lambda \in \mathrm{C}_{\Gamma}$ such that $\mathrm{d}_{2}(\mathrm{x})=\lambda \alpha \mathrm{d}_{1}(\mathrm{x})$ for all $\alpha \in \Gamma$, where $\mathrm{C}_{\Gamma}$ is the extended centroid of $\mathrm{V}$.

Proof: Let $\mathrm{z} \in \mathrm{V}$. Replacing y by $\mathrm{y}+\mathrm{z}$ in (3), we get

$\mathrm{d}_{1}(\mathrm{x}) \gamma \mathrm{D}_{2}(\mathrm{y}, \mathrm{z})=\mathrm{d}_{3}(\mathrm{x}) \gamma \mathrm{D}_{4}(\mathrm{y}, \mathrm{z})$

If we replace $z$ by $z \delta y$ In (4), then 
$\mathrm{d}_{1}(\mathrm{x}) \gamma \mathrm{z} \mathrm{k}(\delta) \mathrm{d}_{2}(\mathrm{y})=\mathrm{d}_{3}(\mathrm{x}) \gamma \mathrm{z} \mathrm{k}(\delta) \mathrm{d}_{4}(\mathrm{y})$

If $\mathrm{w} \in \mathrm{V}$ then

$\mathrm{d}_{1}(\mathrm{x}) \gamma \mathrm{z} \mathrm{k}(\alpha) \mathrm{d}_{2}(\mathrm{w})=\mathrm{d}_{3}(\mathrm{x}) \gamma \mathrm{z} \mathrm{k}(\alpha) \mathrm{d}_{4}(\mathrm{w})$

Substituting $z k(\alpha) d_{4}(w)$ for $z$ in (5), we have

$\mathrm{d}_{1}(\mathrm{x}) \gamma \mathrm{z} \mathrm{k}(\alpha) \mathrm{d}_{4}(\mathrm{w}) \mathrm{k}(\delta) \mathrm{d}_{2}(\mathrm{y})=\mathrm{d}_{3}(\mathrm{x}) \gamma \mathrm{z} \mathrm{k}(\alpha) \mathrm{d}_{4}(\mathrm{w}) \mathrm{k}(\delta) \mathrm{d}_{4}(\mathrm{y})$

From (4) and (6), we have

$\mathrm{d}_{1}(\mathrm{x}) \gamma \mathrm{z} \mathrm{k}(\alpha) \mathrm{d}_{4}(\mathrm{w}) \mathrm{k}(\delta) \mathrm{d}_{2}(\mathrm{y})=\mathrm{d}_{1}(\mathrm{x}) \gamma \mathrm{z} \mathrm{k}(\alpha) \mathrm{d}_{2}(\mathrm{w}) \mathrm{k}(\delta) \mathrm{d}_{4}(\mathrm{y})$

$\Rightarrow \mathrm{d}_{1}(\mathrm{x}) \gamma \mathrm{zk}(\alpha)\left(\mathrm{d}_{4}(\mathrm{w}) \mathrm{k}(\delta) \mathrm{d}_{2}(\mathrm{y})-\mathrm{d}_{2}(\mathrm{w}) \mathrm{k}(\delta) \mathrm{d}_{4}(\mathrm{y})\right)=0$

Since $d_{1} \neq 0$ and $V$ is a prime $\Gamma$-Banach algebra. So equation (8) implies that

$\mathrm{d}_{4}(\mathrm{w}) \mathrm{k}(\delta) \mathrm{d}_{2}(\mathrm{y})=\mathrm{d}_{2}(\mathrm{w}) \mathrm{k}(\delta) \mathrm{d}_{4}(\mathrm{y})$

It follows from $\mathrm{d}_{4} \neq 0$ and lemma 3.5 that $\mathrm{d}_{2}(\mathrm{y})=\lambda \alpha \mathrm{d}_{4}$ (y) for some $\lambda \in \mathrm{C}_{\Gamma}$. Hence by (4), we have $\mathrm{d}_{1}(\mathrm{x}) \gamma \mathrm{z}$ $\mathrm{k}(\delta) \lambda \alpha \mathrm{d}_{4}(\mathrm{y})=\mathrm{d}_{3}(\mathrm{x}) \gamma \mathrm{z} \mathrm{k}(\delta) \mathrm{d}_{4}(\mathrm{y})$

Since $\lambda \in \mathrm{C}_{\Gamma}$. Therefore

$\mathrm{d}_{1}(\mathrm{x}) \gamma \mathrm{k}(\delta) \mathrm{d}_{4}(\mathrm{y}) \alpha \lambda=\mathrm{d}_{3}(\mathrm{x}) \gamma \mathrm{z} \mathrm{k}(\delta) \mathrm{d}_{4}(\mathrm{y})$

$\Rightarrow \lambda \alpha \mathrm{d}_{1}(\mathrm{x}) \gamma \mathrm{k}(\delta) \mathrm{d}_{4}(\mathrm{y})=\mathrm{d}_{3}(\mathrm{x}) \gamma \mathrm{z} \mathrm{k}(\delta) \mathrm{d}_{4}(\mathrm{y})$

$\Rightarrow\left[\lambda \alpha \mathrm{d}_{1}(\mathrm{x})-\mathrm{d}_{3}(\mathrm{x})\right] \gamma \mathrm{z} \mathrm{k}(\delta) \mathrm{d}_{4}(\mathrm{y})=0$

It follows from $d_{4} \neq 0$ that $d_{3}(x)=\lambda \alpha d_{1}(x)$.

Lemma3.8. Let $\mathrm{V}$ be a 2-torsion free prime Gamma Banach algebra and let $\mathrm{U}$ be a nonzero ideal of $\mathrm{V}$. Let a, $\mathrm{b} \in \mathrm{V}$ be fixed elements. If $\mathrm{a} \gamma \mathrm{x} \beta \mathrm{b}+\mathrm{b} \gamma \mathrm{x} \beta \mathrm{a}=0$ is fulfilled for all $\mathrm{x} \in \mathrm{U}, \alpha, \beta \in \Gamma$ then either $\mathrm{a}=0$ or $\mathrm{b}=0$.

Theorem3.9. Let $\mathrm{V}$ be a 2-torsion free prime Gamma Banach algebra and $\mathrm{U}$ be a non zero ideal of $\mathrm{V}$. Suppose there exist symmetric bi-k-derivations $D_{1}: V \times V \rightarrow V$ and $D_{2}: V \times V \rightarrow V$ such that

$\mathrm{D}_{1}\left(\mathrm{~d}_{2}(\mathrm{x}), \mathrm{x}\right)=0$

holds for all $\mathrm{x} \in \mathrm{U}$ where $\mathrm{d}_{2}$ denotes the trace of $\mathrm{D}_{2}$. In this case $\mathrm{D}_{1}=0$ or $\mathrm{D}_{2}=0$.

Proof: let $\mathrm{y} \in \mathrm{U}$. Replacing $\mathrm{x}$ by $\mathrm{x}+\mathrm{y}$ in $(9)$ we get

$\mathrm{D}_{1}\left(\mathrm{~d}_{2}(\mathrm{x}), \mathrm{y}\right)+2 \mathrm{D}_{1}\left(\mathrm{D}_{2}(\mathrm{x}, \mathrm{y}), \mathrm{x}\right)+\mathrm{D}_{1}\left(\mathrm{~d}_{2}(\mathrm{y}), \mathrm{x}\right)+2 \mathrm{D}_{1}\left(\mathrm{D}_{2}(\mathrm{x}, \mathrm{y}), \mathrm{y}\right)=0$

substituting $\mathrm{x}$ by $-\mathrm{x}$ in (10) we get,

$\mathrm{D}_{1}\left(\mathrm{~d}_{2}(\mathrm{x}), \mathrm{y}\right)+2 \mathrm{D}_{1}\left(\mathrm{D}_{2}(\mathrm{x}, \mathrm{y}), \mathrm{x}\right)=\mathrm{D}_{1}\left(\mathrm{~d}_{2}(\mathrm{y}), \mathrm{x}\right)+2 \mathrm{D}_{1}\left(\mathrm{D}_{2}(\mathrm{x}, \mathrm{y}), \mathrm{y}\right)$

Comparing (10) and (11) we have

$\mathrm{D}_{1}\left(\mathrm{~d}_{2}(\mathrm{x}), \mathrm{y}\right)+2 \mathrm{D}_{1}\left(\mathrm{D}_{2}(\mathrm{x}, \mathrm{y}), \mathrm{x}\right)=0$

Let us replace in (12) y by x $\alpha y, \alpha \in \Gamma$ and use (10) and (12) we get,

$\mathrm{d}_{2}(\mathrm{x}) \mathrm{k}(\alpha) \mathrm{D}_{1}(\mathrm{y}, \mathrm{x})+\mathrm{d}_{1}(\mathrm{x}) \mathrm{k}(\alpha) \mathrm{D}_{2}(\mathrm{x}, \mathrm{y})=0$

Let us write in (13) $\mathrm{y} \beta \mathrm{x}$ instead of $\mathrm{y}$ we have

$\mathrm{d}_{2}(\mathrm{x}) \mathrm{k}(\alpha) \operatorname{vk}(\beta) \mathrm{d}_{1}(\mathrm{x})+\mathrm{d}_{1}(\mathrm{x}) \mathrm{k}(\alpha) \operatorname{vk}(\beta) \mathrm{d}_{2}(\mathrm{x})=0$

Let $d_{1} \neq 0, d_{2} \neq 0$. Then there exist elements $x_{1}, x_{2} \in U$ such that $d_{1}\left(x_{1}\right) \neq 0$ and $d_{2}\left(x_{2}\right) \neq 0$. From (14) and lemma3.8 it follows that $\mathrm{d}_{1}\left(\mathrm{x}_{2}\right)=\mathrm{d}_{2}\left(\mathrm{x}_{1}\right)=0$. Since $\mathrm{d}_{1}\left(\mathrm{x}_{2}\right)=0$ the relation (13) reduces to $d_{2}\left(x_{2}\right) k(\alpha) D_{1}\left(y, x_{2}\right)=0$. Using this relation and lemma3.5, we obtain that $\mathrm{D}_{1}\left(\mathrm{y}, \mathrm{x}_{2}\right)=0$ holds for all $\mathrm{y} \in \mathrm{U}$ since $\mathrm{d}_{2}\left(\mathrm{x}_{2}\right) \neq 0$. In particular, we have $\mathrm{D}_{1}\left(\mathrm{x}_{1}, \mathrm{x}_{2}\right)=0$ and so

$\mathrm{d}_{1}\left(\mathrm{x}_{1}+\mathrm{x}_{2}\right)=\mathrm{d}_{1}\left(\mathrm{x}_{1}\right)+\mathrm{d}_{1}\left(\mathrm{x}_{2}\right)+2 \mathrm{D}_{1}\left(\mathrm{x}_{1}, \mathrm{x}_{2}\right)$

$=\mathrm{d}_{1}\left(\mathrm{x}_{1}\right) \neq 0$

Similarly we obtain $\mathrm{d}_{2}(\mathrm{y}) \neq 0$. But $\mathrm{d}_{1}(\mathrm{y})$ and $\mathrm{d}_{2}(\mathrm{y})$ cannot be both different from zero according to (14) and lemma 3.8. Therefore we have either $\mathrm{d}_{1}=0$ or $\mathrm{d}_{2}=0$. 
Corollary3.10. Let $\mathrm{V}$ be a 2-torsion free semi-prime gamma banach algebra and $\mathrm{U}$ be non zero ideal of $\mathrm{V}$. Suppose there exists a symmetric bi-k-derivation $\mathrm{D}: \mathrm{V} \times \mathrm{V} \rightarrow \mathrm{V}$ such that $\mathrm{D}(\mathrm{d}(\mathrm{x}), \mathrm{x})=0$ holds for all $\mathrm{x} \in \mathrm{U}$, where $\mathrm{d}$ denotes the trace of $\mathrm{D}$. In this case we have $\mathrm{d}=0$.

Theorem3.11. Let $\mathrm{V}$ be a 2- and 3-torsion free prime $\Gamma$-Banach algebra. Let $\mathrm{U}$ be a non zero ideal of $\mathrm{V}$ and $D_{1}: V \times V \rightarrow V$ and $D_{2}: V \times V \rightarrow V$ be symmetric bi-k- derivations. Suppose further that there exists a symmetric bi-additive mapping $\mathrm{B}: \mathrm{V} \times \mathrm{V} \rightarrow \mathrm{V}$ such that $\mathrm{d}_{1}\left(\mathrm{~d}_{2}(\mathrm{x})\right)=\mathrm{f}(\mathrm{x})$ holds, for all $\mathrm{x} \in \mathrm{U}$, where $d_{1}$ and $d_{2}$ are the traces of $\mathrm{D}_{1}$ and $\mathrm{D}_{2}$ respectively and $\mathrm{f}$ is the trace of $\mathrm{B}$. Then either $\mathrm{D}_{1}=0$ and $\mathrm{D}_{2}=0$.

Proof: Putting $x+y$ in place of $x$ in $d_{1}\left(d_{2}(x)\right)=f(x)$, we get

$2 \mathrm{~d}_{1}\left(\mathrm{D}_{2}(\mathrm{x}, \mathrm{y})\right)+\mathrm{D}_{1}\left(\left(\mathrm{~d}_{2}(\mathrm{x}), \mathrm{d}_{2}(\mathrm{y})\right)+2 \mathrm{D}_{1}\left(\mathrm{~d}_{2}(\mathrm{x}), \mathrm{D}_{2}(\mathrm{x}, \mathrm{y})\right)\right.$

$+2 \mathrm{D}_{1}\left(\mathrm{~d}_{2}(\mathrm{y}), \mathrm{D}_{2}(\mathrm{x}, \mathrm{y})\right)=\mathrm{B}(\mathrm{x}, \mathrm{y})$

Let us replace in (15) $\mathrm{x}$ by $-\mathrm{x}$. We have

$2 \mathrm{~d}_{1}\left(\mathrm{D}_{2}(\mathrm{x}, \mathrm{y})\right)+\mathrm{D}_{1}\left(\left(\mathrm{~d}_{2}(\mathrm{x}), \mathrm{d}_{2}(\mathrm{y})\right)-2 \mathrm{D}_{1}\left(\mathrm{~d}_{2}(\mathrm{x}), \mathrm{D}_{2}(\mathrm{x}, \mathrm{y})\right)\right.$

$-2 \mathrm{D}_{1}\left(\mathrm{~d}_{2}(\mathrm{y}), \mathrm{D}_{2}(\mathrm{x}, \mathrm{y})\right)=-\mathrm{B}(\mathrm{x}, \mathrm{y})$

Adding (15) and (16) we get,

$2 \mathrm{~d}_{1}\left(\mathrm{D}_{2}(\mathrm{x}, \mathrm{y})\right)+\mathrm{D}_{1}\left(\left(\mathrm{~d}_{2}(\mathrm{x}), \mathrm{d}_{2}(\mathrm{y})\right)=0\right.$

Then equation (15) reduces

$2 \mathrm{D}_{1}\left(\mathrm{~d}_{2}(\mathrm{x}), \mathrm{D}_{2}(\mathrm{x}, \mathrm{y})\right)+2 \mathrm{D}_{1}\left(\mathrm{~d}_{2}(\mathrm{y}), \mathrm{D}_{2}(\mathrm{x}, \mathrm{y})\right)=\mathrm{B}(\mathrm{x}, \mathrm{y})$

Let us write in (17) $2 \mathrm{x}$ instead of $\mathrm{x}$. we get

$$
8 D_{1}\left(d_{2}(x), D_{2}(x, y)\right)+2 \mathrm{D}_{1}\left(\mathrm{~d}_{2}(\mathrm{y}), \mathrm{D}_{2}(\mathrm{x}, \mathrm{y})\right)=\mathrm{B}(\mathrm{x}, \mathrm{y})
$$

Now substitute (17) from (18) we get

${ }_{6} \mathrm{D}_{1}\left(\mathrm{~d}_{2}(\mathrm{x}), \mathrm{D}_{2}(\mathrm{x}, \mathrm{y})\right)=0$

$\Rightarrow \mathrm{D}_{1}\left(\mathrm{~d}_{2}(\mathrm{x}), \mathrm{D}_{2}(\mathrm{x}, \mathrm{y})\right)=0$

Since V is a 2- and 3-torsion free Gamma Banach algebra. It follows that both terms on the left side of relation (17) are zero, which means that $\mathrm{B}=0$.Then

$\mathrm{d}_{1}\left(\mathrm{~d}_{2}(\mathrm{x})\right)=0, \mathrm{x} \in \mathrm{U}$.

Substituting yox, $\alpha \in \Gamma$ for $\mathrm{y}$ in (19) we get

$\mathrm{D}_{1}\left(\mathrm{~d}_{2}(\mathrm{x}), \mathrm{y}\right) \mathrm{k}(\alpha) \mathrm{d}_{2}(\mathrm{x})+\mathrm{D}_{2}(\mathrm{x}, \mathrm{y}) \mathrm{k}(\alpha) \mathrm{D}_{1}\left(\mathrm{~d}_{2}(\mathrm{x}), \mathrm{x}\right)=0$

Let us write $\mathrm{x} \beta \mathrm{y}, \beta \in \Gamma$ instead of $\mathrm{y}$ we have

$D_{1}\left(d_{2}(x), x\right) k(\beta) y k(\alpha) d_{2}(x)+d_{2}(x) k(\beta) y k(\alpha) D_{1}\left(d_{2}(x), x\right)=0$

From the relation above one can conclude that

$\mathrm{D}_{1}\left(\mathrm{~d}_{2}(\mathrm{x}), \mathrm{x}\right)=0$ or $\mathrm{d}_{2}(\mathrm{x})=0$

If $\mathrm{D}_{1}\left(\mathrm{~d}_{2}(\mathrm{x}), \mathrm{x}\right) \neq 0$ for some $\mathrm{x} \in \mathrm{U}$, then $\mathrm{d}_{2}(\mathrm{x})=0$

Contrary to the assumption $\mathrm{D}_{1}\left(\mathrm{~d}_{2}(\mathrm{x}), \mathrm{x}\right) \neq 0$

There $\mathrm{D}_{1}\left(\mathrm{~d}_{2}(\mathrm{x}), \mathrm{x}\right)=0$, for all $\mathrm{x} \in \mathrm{U}$, the proof of the theorem is complete since all the requirements of theorem 3.10 are fulfilled.

Corollary3.12 Let $\mathrm{V}$ be a semi-prime gamma ring which is 2-and 3-torsion free. Let $\mathrm{U}$ be a nonzero ideal of $\mathrm{V}$ and $d(U) \subset U$. Let $D: V \times V \rightarrow V$ be a symmetric bi-k-derivation and $B: V \times V \rightarrow V$ be a symmetric bi-additive mapping. Suppose that $d(d(x))=f(x)$ holds for all $x \in U$, where $d$ is the trace of $D$ and $f$ is the trace of $B$. In this case we have $\mathrm{D}=0$.

Example2. Let $V=\left\{\left(\begin{array}{ll}x & 0 \\ 0 & 0\end{array}\right): x \in R\right\}$ and $\Gamma=\left\{\left(\begin{array}{ll}\mathrm{n} & 0 \\ 0 & \mathrm{n}\end{array}\right): \mathrm{x} \in \mathrm{R}\right\}$

Then $\mathrm{V}(\mathrm{F}=\mathrm{R})$ is a $\Gamma$-Banach algebra. 
$\mathrm{D}: \mathrm{V} \times \mathrm{V} \rightarrow \mathrm{V}$ define as $\mathrm{D}(\mathrm{A}, \mathrm{B})=\mathrm{AB}, \quad$ for all $\mathrm{A}, \mathrm{B} \in \mathrm{V}$ and $\mathrm{k}: \Gamma \rightarrow \Gamma$ define as $\mathrm{k}(\alpha)=\frac{1}{\mathrm{n}} \alpha$, where $\alpha=\left\{\left(\begin{array}{cc}n & 0 \\ 0 & n\end{array}\right) \in \Gamma\right\}$. Then it is easy to show that $\mathrm{D}$ is a symmetric bi-k-derivation.

\section{References:}

[1] C.Selvaraj and S.Petchimuthu, On strongly Prime Gamma Rings, International J. of Algebra, 2(2008), $933-943$.

[2] D. K. Bhattacharya and A. K. Maity, Semilinear tensor product of Gamma Banach Algebras, Ganita,140(1989), 75-80. H.Kandamar, The k-derivation of a Gamma-Ring, Jurk.J. Math, 24 (2000), 221-231.

J. Luh, On the theory of simple Gamma rings,Michigan Math. 16(1969), 65-75.

M. Ali Ozturk and y-Imaz Ceven, On $(\sigma, \tau)$-Gamma-Derivations in Gamma-Near-Rings, Advances in Algebra, 1(2008),1-10

M. Ali Ozturk and Young Bae Jun, On the Centroid of the prime Gamma rings, Comm. Korean Math. Soc., 15 (2000), 469-479.

N. Nobusawa, on a generalization of the ring theory, Osaka J. Math. 1(1964), 81-89.

[8] N.B.Okelo, M.O. Okongo and S.A. Nyoukiti, On projective Tensor Norm and Norm-Attainable $\alpha$ - derivation, Int. J.Contemp. Math. Sciences, 5 (2010), 1969-1975.

[9] S.Chakraborty and A.Ch. paul, On Jordan k-derivation of 2-torsion free prime $\Gamma_{\mathrm{N}}$-Rings, Journal of Mathematics, 40 (2008), 97-101

[10] S.Kyuno, On prime Gamma Rings, Pacific J. of Mathematics, 75(1978), 185-190.

[11] W. E. Barnes, On the rings of Nobusawa, Pacific J. Math. 18(1966), 411-422.

[12] Y.Ceven and M.Ali Qzturk, On Jordan Generalized Derivations in gamma rings, Hacettepe Journal of Mathematics and statistic, 33(2004), 11-14. 